\title{
Cuidado de enfermería a un paciente con Diabetes Mellitus. Estudio de caso.
}

\author{
Guevara-Valtier Milton Carlos ${ }^{1, c}$, Santos-Flores Jesús Melchor ${ }^{1, e}$, \\ Paz-Morales María de los Ángeles ${ }^{1, f}$, Gutiérrez-Valverde Juana Mercedes ${ }^{1, a}$.
}

\begin{abstract}
RESUMEN
En México la obesidad y la Diabetes Mellitus tipo 2 (DM2) ocupan los primeros lugares de morbilidad y mortalidad, el tratamiento de estas enfermedades suele ser complejo e involucra una serie de actividades colaborativas con el equipo multiprofesional en el que sin duda el rol de enfermería es pieza fundamental para el logro de resultados esperados. Objetivo: brindar cuidado de especializado de enfermería con base al PAE para el control del paciente con DM2. Presentación del Caso (Evidencias, Diagnósticos), el caso clínico se guío mediante el Proceso de Atención de Enfermería (Valoración, Diagnóstico, Plan de Intervenciones, Ejecución y Evaluación) en un hospital público ubicado en Monterrey, México. Evaluación: se lograron 8 de 9 objetivos plateados. Conclusiones: logró satisfactorio de objetivos planteados, un $88,88 \%$ de los resultados esperados fueron conseguidos.
\end{abstract}

PALABRAS CLAVE: Obesidad, diabetes mellitus, enfermedad crónica.

\section{Running a nursing process for a patient with obesity and diabetes mellitus type 2}

\section{SUMMARY}

In Mexico the obesity and Diabetes Mellitus Type 2 (DM2) taking up the first places of morbidity and mortality, the treatment of these diseases usually are complex and involve a series of collaborative activities with the multi-professional in which certainly the nursing role is a fundamental piece for achieving of expected results. Objectives: To bring the care of specialized of nursing based in the PAE for the patient's control with DM2. Case Report: the clinic case was guided by means of The Attention Process of Nursing (Valuation, Diagnosis, Intervention Plan, Execution, and Evaluation) in a public hospital located in Monterrey, Mexico. Evaluation: they were accomplished 8 of 9 planned objectives. Conclusions: it achieved satisfactorily of the planned objectives, the $88,88 \%$ of the expected results and they were well made.

KEY WORDS: Obesity, diabetes mellitus, chronic disease.

Facultad de Enfermería, Universidad Autónoma de Nuevo León. Nuevo León, México.

Hospital Universitario, Universidad Autónoma de Nuevo León. Nuevo León, México.

Doctorado en Ciencias de Enfermería, ${ }^{b}$ Maestría en Ciencias de Enfermería, ${ }^{c}$ Doctorado en Educación, ${ }^{\mathrm{d}}$ E. Licenciado en Enfermería,

E. Licenciado en Enfermería, ${ }^{\mathrm{f}}$ Doctorado en Educación. 


\section{INTRODUCCIÓN}

La diabetes mellitus tipo 2 constituye un problema en salud redundante porque es una de las enfermedades crónicas más frecuentes, la cual causa varias complicaciones agudas (cetoacidosis, hiperglicemia, hipoglucemia) y crónicas (retinopatía, nefropatía, neuropatía, enfermedad isquémica del corazón) disminuyendo la calidad de vida. De acuerdo con la Encuesta Nacional de Salud y Nutrición 2012(ENSANUT)(1), hay cerca de 6,5 millones de personas que refieren haber sido diagnosticadas con diabetes.

Arriaga menciona que "la aplicación del método científico en la práctica asistencial enfermera, es el método conocido como PAE. Este método permite a enfermería prestar cuidados de una forma racional, lógica y sistemática" en este sentido permite y facilita el diseño de las mejores intervenciones para favorecer el cuidado, control y mejora significativa en la calidad de vida de los individuos, familias y comunidades (1).

\section{Presentación del caso (Evidencias y diagnósticos).}

\section{Valoración:}

Paciente masculino de 43 años de edad,procedente de Monterrey Nuevo León, México, estado civil casado, internado en el Hospital Universitario. Dr. José Eleuterio González, ingresa el 7 de Diciembre de 2015, en el área de Medicina Interna.

\section{Tratamiento e indicaciones actuales:}

dieta diabética, dextrosis postprandial, administración de medicamentos Ciprofloxacina $400 \mathrm{mg} \mathrm{I.V}$. cada 12 horas, Ketorolaco $30 \mathrm{mg}$ I.V. cada 8 horas y Captopril $25 \mathrm{mg}$ V.O. cada 12 horas.

Antecedentes familiares: madre con antecedentes de diabetes mellitus tipo 2 .

Antecedentes patológicos personales: obesidad diagnosticada a la edad de 20 años.

Hipertensión arterial: diagnosticada 5 años antes de su ingreso, en tratamiento con Captopril $25 \mathrm{mg}$ cada 12 horas. Diabetes Mellitus tipo II: desde los 20 años de edad, utiliza Insulina N.P.H.

Extracción quirúrgica de ojo derecho a los 7 años de edad.

\section{Exploración fisica:}

con biotipo bravilíneo y constitución endomórfica.

Signos vitales y somatometria, Temperatura: $36,9^{\circ} \mathrm{C}$,
Presión arterial: 160/80 mmHg, Pulso: 87x', Frecuencia respiratoria: 26x', Peso actual: $98 \mathrm{Kg}$, Talla: $1.69 \mathrm{mts}$, IMC: 34,31 .

Piel: color morena, tibia, presenta buena hidratación, textura suave con buena higiene. Lesión secundaria por catéter periférico en el antebrazo izquierdo. Con intervención quirúrgica en pie izquierdo. Cráneo: cráneo normocéfalo, forma redonda, proporcional al cuerpo.

Cabello: color negro, cantidad moderada, buena higiene. Correcta implantación y distribuido uniformemente.

Cara: forma redonda, simétrica a sus componentes, piel color morena.

Ojos: cejas abundantes de color negro, simétricas a su cara, parpados y pestañasíntegros, globo ocular izquierdo proporcional a su rostro. Iris color café claro, con buena vascularización, pupila reactiva y con reflejo, miosis y midriasis presente. Conjuntiva con buena hidratación. Esclerótica color blanca, campo visual izquierdo de 170 grados. Ausencia de ojo derecho.

Oídos: forma ovalada tamaño mediano con buena simetría, pabellones auriculares simétricos, conductos auditivos de ambos oídos permeables, cuenta con escaso cerumen. Con buena agudeza auditiva.

Nariz: redonda, alineada sin presencia de aleteo nasal, permeable,mucosa rosada.

Boca: ausencia de incisivo inferior derecho. Mucosa, paladar y encías rosadas, sin dolor, no presenta gingivitis, amígdalas rosadas y sin inflamación.

Cuello: corto y grueso, cuenta con movilidad, ganglios linfáticos ausentes, glándula tiroidea sin dolor.Tórax: forma hiposténico, simétrico, correcta retracción de espacio intercostal, columna alineada. Expansión torácica presente, con amplexion y amplexacion presentes, frémito vocal presente no se escucha disminuido.

Abdomen: blando, depresible, no doloroso. peristaltismo presente.

Extremidades superiores: con simetría correcta, cuenta con movimientos presentes.

Extremidades inferiores: cuenta con movimientos en pie derecho y ausente en izquierdo, con zonas de dolor en pie izquierdo por la intervención quirúrgica.

Genitales: genitales íntegros, sin masas anormales, sin zonas con dolor.

\section{Exámenes auxiliares:}

biometría hemática: eritrocitos $3.80 \mathrm{M} / \mathrm{uL}$, hemoglobina $11.7 \mathrm{~g} / \mathrm{dL}$, hematocrito $35.1 \%$, leucocitos $13,0 \mathrm{~K} / \mathrm{uL}$, Neutrófilos $9.10 \mathrm{~K} / \mathrm{uL}$.

Química sanguínea: glucosa en sangre $157 \mathrm{mg} / \mathrm{dl}$, creatinina sérica $1,5 \mathrm{mg} / \mathrm{dl}$, nitrógeno de la urea en sangre $21 \mathrm{mg} / \mathrm{dl}$. 


\section{Diagnóstico:}

con la utilización de Nursing Diagnoses Definitions and Classification NANDA 2015-2017(2) se lograron encontrar los diagnósticos de enfermería pertinentes para el caso (Tabla 1).

\section{Planeación:}

Con respecto a los diagnósticos identificados se planteó un plan de intervenciones considerando como modelo la Clasificación de Intervenciones de Enfermería (3) y la Clasificación de Resultados de Enfermería(4), el plan se ajustó a las necesidades individuales del usuario con Obesidad y DM2, en conjunto con esto se pensó y redactó una serie de resultados esperados en conjunto con indicadores de evaluación con el fin de facilitar la evaluación del mismo, la propuesta se presenta (tabla 2).

Cabe aclarar que además de seguir el plan propuesto se consideró la inclusión de las metas y estándares internacionales (Meta No 1.- Identificar correctamente a los pacientes, Meta No 2.-Mejorar la comunicación efectiva, Meta No 5.- Reducir el riesgo de infecciones asociadas a la atención médica, Meta No 6.- Reducir el Riesgo de Daño al paciente por causa de caídas, acceso y continuidad de la atención, derechos de los pacientes y de su familia, evaluación de pacientes, atención de pacientes, manejo y uso de medicamentos, educación del paciente y de su familia, mejora de la calidad y seguridad del paciente, prevención y control de infecciones) (5), así como recomendaciones de aplicabilidad institucional relacionadas con la calidad y seguridad del paciente.

\section{Ejecución}

Considerando los recursos disponibles de tiempo, espacio, insumos y aspectos individuales del paciente y su familia se ejecutó el plan de acciones para el logro de las actividades (Tabla 3), en este sentido el plan propuesto y las acciones ejecutadas fueron apoyadas con las indicaciones establecidas en los Planes de Atención de Enfermería diseñados para ser ejecutados en el ámbito nacional mexicano $(6,7)$.

\section{Evaluación}

El plan de atención demostró estimular de forma positiva cada uno de los resultados propuestos.

El 88,88\% de los resultados esperados fueron logrados. Se logró un control del riesgo de nivel de glucemia inestable mediante la vigilancia de la glucemia con la realización de glicemia capilar y aplicación de insulina, manteniendo los niveles óptimos de glucosa en sangre.

El diagnóstico dolor agudo se priorizo tomando en cuenta que si no se trata de manera adecuada puede tener múltiples consecuencias, un incremento en la estancia hospitalaria, aumento de los costos, insatisfacción del paciente, no solo se incluyó la administración de analgésicos que en este caso se administró ketorolaco $30 \mathrm{mg}$; sino que

Tabla 1. Modelo de Análisis y Priorización

\begin{tabular}{|c|c|}
\hline Datos relevantes (DR) & Diagnósticos de Enfermería (Dx) \\
\hline $\begin{array}{l}\text { 1. Refiere cansancio. } \\
\text { 2. Peso actual es de } 98 \text { kilogramos. } \\
\text { 3. Micciona siete veces al día. } \\
\text { 4. Movimientos no coordinados en pierna izquierda. } \\
\text { 5. Ausencia de ojo derecho. } \\
\text { 6. Amputación de pierna izquierda. } \\
\text { 7. Antecedentes familiares de Diabetes mellitus tipo } 2 . \\
\text { 8. Diabetes mellitus tipo } 2 \text { desde los } 20 \text { años. } \\
\text { 9. Presenta marcha disbasica. } \\
\text { 10. Cambio de patrón de sueño (disminución de } 8 \text { a } 4 \text { horas). } \\
\text { 11. Refiere sentir dolor en el área de la intervención } \\
\text { quirúrgica. } \\
\text { 12. Edad de } 43 \text { años. } \\
\text { 13. Sexo masculino. } \\
\text { 14. Vida sedentaria. } \\
\text { 15. Dieta diaria a base de carbohidratos } \\
\text { 16. Consume platillos de mas de } 4000 \text { kilocalorias }\end{array}$ & $\begin{array}{l}\text { 1. Riesgo de nivel de glucemia inestable } \\
\text { (DR:1,2,7,8,13,14,15). } \\
\text { 2. Riesgo de caídas (DR:2,4,5,6,9). } \\
\text { 3. Riesgo de infección (DR:8). } \\
\text { 4. Trastorno del patrón del sueño (DR:10). } \\
\text { 5. Desequilibrio nutricional: Ingesta superior a las necesida- } \\
\text { des (DR:2,13,14,15,16). }\end{array}$ \\
\hline
\end{tabular}


Tabla 2. El esquema para el resultado e intervención

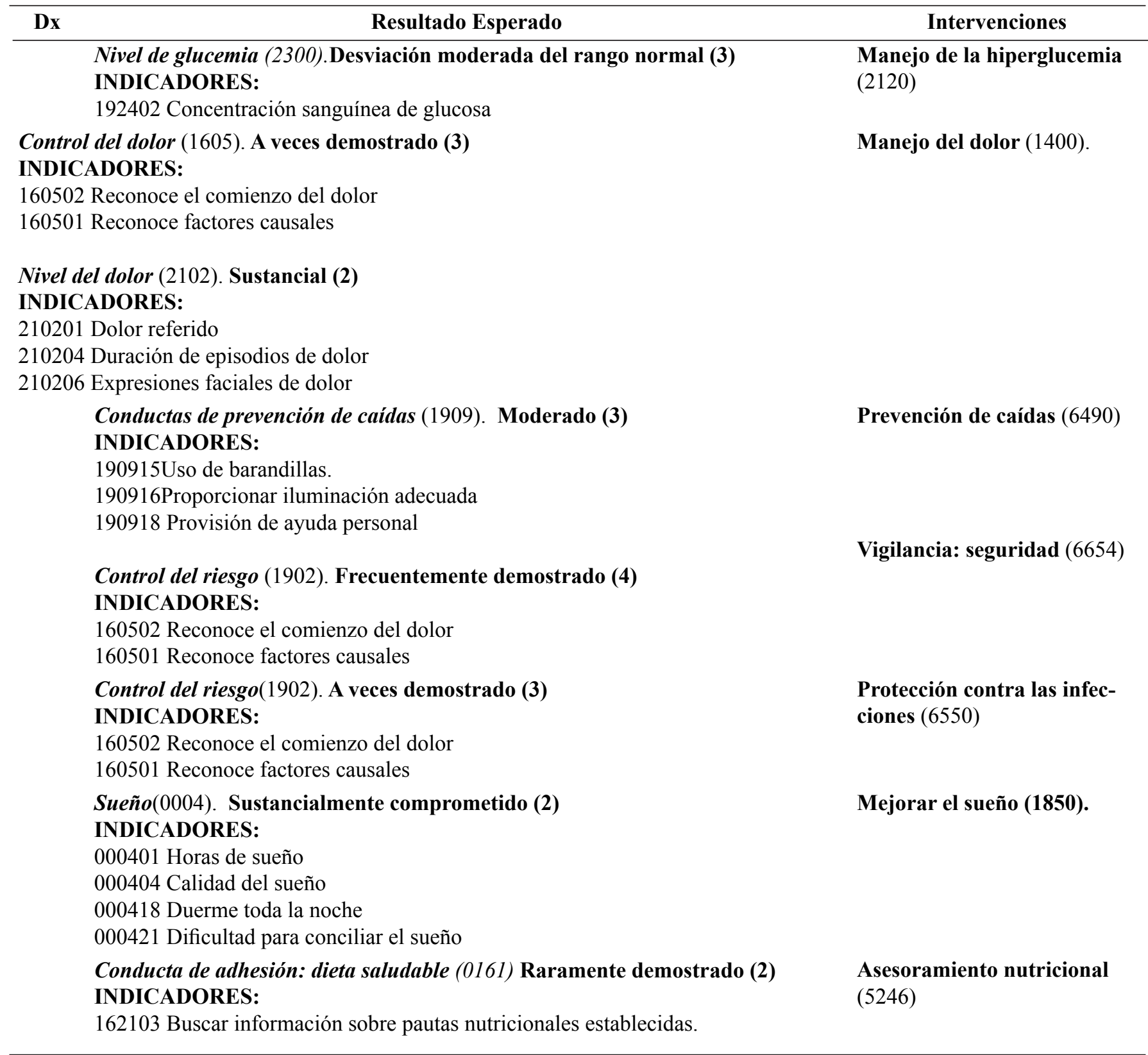

también fue necesario la incorporación del paciente y su familia para que con su colaboración activa con el equipo de salud el alivio del dolor sea más satisfactorio y efectivo, se utilizaron además coadyuvantes no farmacológicas como la relajación (8).

El riesgo de caídas fue disminuido, mediante métodos de enseñanza sobre factores de riesgo que podrían desencadenar caídas, se utilizaron materiales que se encontraban al alcance para prevenir el riesgo como por ejemplo utilizar las barandillas de manera correcta y mantener una ilumi- nación adecuada. El riesgo de infección fue disminuido mediante la aplicación de antibioticoterapia se administró ciprofloxacino $400 \mathrm{mg}$ y mantenimiento un ambiente seguro y limpio.

Trastorno del patrón del sueño fue logrado mediante la modificación del entorno, iluminación, ruido, control del dolor. En el déficit de autocuidados: uso del inodoro, no se logró el objetivo, sin embargo, logro evacuar satisfactoriamente gracias al uso de un bidel. 
Tabla 3. Plan de acciones para el logro de las actividades

\section{INTERVENCIÓN}

Manejo de la hiperglucemia (2120)

1.- Vigilar los niveles de glucosa en sangre, si está indicado.

2.- Administrar insulina, según prescripción médica.

3.- Mantener una vía i.v., si lo requiere el caso.

Manejo del dolor (1400)

4.- Realizar la valoración del dolor según escala.

5.- Observar claves no verbales de molestias.

6.- Seleccionar y desarrollar aquellas medidas (farmacológica, no farmacológica e interpersonal) que faciliten el alivio del dolor, si procede.

Prevención de caídas (6490)

7.- Identificar déficit cognoscitivo o físico del paciente que puedan aumentar la posibilidad de caídas en un ambiente dado.

Vigilancia: seguridad (6654)

8.- Utilizar barandillas laterales de longitud y altura adecuadas para evitar caídas de la cama, si es necesario.

Protección contra las infecciones (6550)

9.- Inspeccionar la existencia de enrojecimiento, calor extremo o drenaje en la piel y las membranas mucosas.

10.- Inspeccionar el estado de cualquier incisión/herida quirúrgica.

Mejorar el sueño (1850)

11.- Ajustar el ambiente (luz, ruido, temperatura, colchón y cama) para favorecer el sueño.

\section{Asesoramiento nutricional (5246)}

14.- Determinar la ingesta y los hábitos alimentarios del paciente.

15.- Proporcionar información acerca de las necesidades de modificación de la dieta por razones de salud: pérdida de peso.

\section{Resultado Esperado}

Nivel de glucemia (2300).

Desviación moderada del rango normal (3)

Control del dolor (1605).

A veces demostrado (3)

Nivel del dolor (2102).

Sustancial (2)

Conductas de prevención de caídas (1909).

Moderado (3)

Control del riesgo (1902).

Frecuentemente demostrado (4)

Control del riesgo (1902).

A veces demostrado (3)

Sueño (0004).

Sustancialmente comprometido (2)

Autocuidados: uso del inodoro(0310)

Sustancialmente comprometido (2)

Conducta de adhesión: dieta saludable (0161) Raramente demostrado (2)

\section{Nivel del logro obtenido}

Sin desviación del rango normal (5)

Siempre demostrado (5)

Ninguno (5)

Ninguno (5)

Siempre demostrado (5)

Siempre demostrado (5)

No comprometido (5)

Levemente comprometido (4)

Siempre demostrado (5)

\section{DISCUSIÓN}

La pérdida de peso permite una mejoría significativa en la sensibilidad a la insulina, la glucosa en ayuno y la concentración de los triglicéridos. Una pérdida de peso de alrededor de $5 \mathrm{~kg}$ se asocia con una reducción de la presión arterial sistólica (PAS) de entre 3,8-4,4 mmHg y una reducción de la presión arterial diastólica (PAD) de 
entre 3,0-3,6 mmHg a los 12 meses. Los pacientes con diabetes mellitus tipo 2 que tienen una pérdida de peso de $5 \mathrm{~kg}$, muestran una reducción de la glucosa plasmática de ayuno de entre $3.06 \mathrm{mg}$ a 4,32 $\mathrm{mg}$ a los 12 meses. Una pérdida de peso alrededor de $5 \mathrm{~kg}$ en pacientes obesos con diabetes tipo 2 se asocia con una reducción de la HbAlc de alrededor de $0,28 \%$ a los 12 meses (9).

Informar y realizar intervenciones de asesoramiento a los pacientes sobre los beneficios asociados con la pérdida de peso hay una mejoría del perfil de lípidos, disminución de la discapacidad asociada con osteoartrosis, disminución de la mortalidad por todas las causas, cáncer y diabetes mellitus, disminución de la presión arterial, mejoría del control de la glucosa (9).

El control estricto de la glucemia así como de la presión arterial, es indispensable para evitar complicaciones y disminuir la mortalidad (10).

\section{CONCLUSIONES}

La participación de enfermería en la atención del paciente con obesidad y diabetes mellitus tipo 2 junto con el personal multidisciplinario es de suma importancia. Las estrategias implementadas para modificar el estilo de vida y favorecer el autocuidado, como herramientas que le permitan al equipo multidisciplinario de salud tomar la mejor decisión clínica para alcanzar las metas de control glucémico y metabólico reflejándose en una atención médica sistematizada (10).

El asesoramiento nutricional, disminuirá las complicaciones futuras a largo plazo ocasionadas por la Diabetes Mellitus tipo 2 e Hipertensión Arterial, mejorando los hábitos alimentarios, lo cual debe ir acompañado de un incremento de la actividad física es el primer paso para el tratamiento de la obesidad en el adulto (9).

Objetivos logrados: en un $88,88 \%$, otorgando una atención de calidad al paciente, incorporando estrategias mediante intervenciones y resultados permitiendo de esta manera aumentar la satisfacción en sus necesidades, ofreciendo y apoyando en su control tanto de obesidad como diabetes mellitus tipo 2 .

\section{REFERENCIAS BIBLIOGRÁFICAS}

1. Arriaga M, Baca J, Castaño C, Montoya M. (eds.): Desde Andalucía: Mujeres del Mediterraneo. Sevilla: Arcibel; 2006.p. 225-226.

2. Heardman TH, Kamitsuru S. Nanda International Nursing
Diagnoses: Definitions \& Classification, 2015-2017. Oxford: Wiley Blackwell; 2014.

3. Bulechek MG, Butchek KH, McCloskey DJ. Clasificación de Intervenciones de Enfermería (NIC). $5^{\circ}$ ed. Madrid: Editorial Elsevier Mosby; 2009

4. Moorhead S, Johnson M, Maas LM, Swanson E. Clasificación de resultados de enfermeria. Cuarta edición. Madrid: Mosby; 2009

5. Consejo de Salubridad General. Estándares para la Certificación de Hospitales 2012. México DF: Consejo de Salubridad General; 2012.p. 27-207.

6. Gobierno de Jalísco, Secretaría de Salud. Plan de Cuidados de Enfermería Estandarizado "Place" Para Pacientes con Obesidad. México DF: Gobierno de Jalísco, Secretaría de Salud; 10-69.

7. Gobierno de Jalísco, Secretaría de Salud. Plan de Cuidados de Enfermería Estandarizado "Place" Para Pacientes con Diabetes Mellitus. México DF: Gobierno de Jalísco, Secretaría de Salud; 10-50.

8. Moreno MMG. Fundamentos para el manejo del dolor posoperatorio "Gestión de enfermería". México DF: Manual Moderno; 2015 .

9. Centro Nacional de Excelencia Tecnológica en Salud. GPC Prevención, Diagnóstico y Tratamiento del sobrepeso y la obesidad exógena. México DF: Centro Nacional de Excelencia Tecnológica en Salud; 2008.

10. Centro Nacional de Excelencia Tecnológica en Salud. GPC Tratamiento de la Diabetes Mellitus Tipo 2. México DF: Centro Nacional de Excelencia Tecnológica en Salud; 2014.

\section{Correspondencia:}

Milton Carlos Guevara Valtier

Correo electrónico carlos_valtier7@hotmail.com

Fecha de Recepción: 27 de noviembre del 2015

Fecha de aceptación: 10 de diciembre del 2015 